\title{
Battle over eye medicine gives drugmaker a dose of reality
}

The drug Avastin has made headlines in recent years for its ability to treat colon and lung cancers. But when the South San Franciscobased biotechnology company Genentech, which developed Avastin, revealed plans in October 2007 to place certain limitations on the distribution of the drug, it was eye doctors who cried foul.

On 20 December, after enduring criticism from retinal specialists, the company amended its decision to restrict sales of the medication. Genetech announced that it would not block doctors' access to the drug. Still, the ordeal has left lingering concerns about the availability of Avastin, and has raised larger questions about drug pricing.

The power of Avastin, a synthetic antibody molecule, lies in its ability to limit blood vessel growth. It was approved by the US Food and Drug Administration for the treatment of colon cancer in 2004. But eye doctors found that in small doses-available for \$15-50 from compounding pharmacies-Avastin also worked better than anything else available for conditions such as "wet" macular degeneration, a disorder in which abnormal blood vessel development in the eye can lead to blindness. The age-related form of this disease strikes approximately 200,000 people each year in the United States alone.

Genentech quickly developed Lucentis-a truncated version of the same antibody used to create Avastin-and tested it specifically for the eye. Approved in 2006 to treat certain forms of macular degeneration, Lucentis was

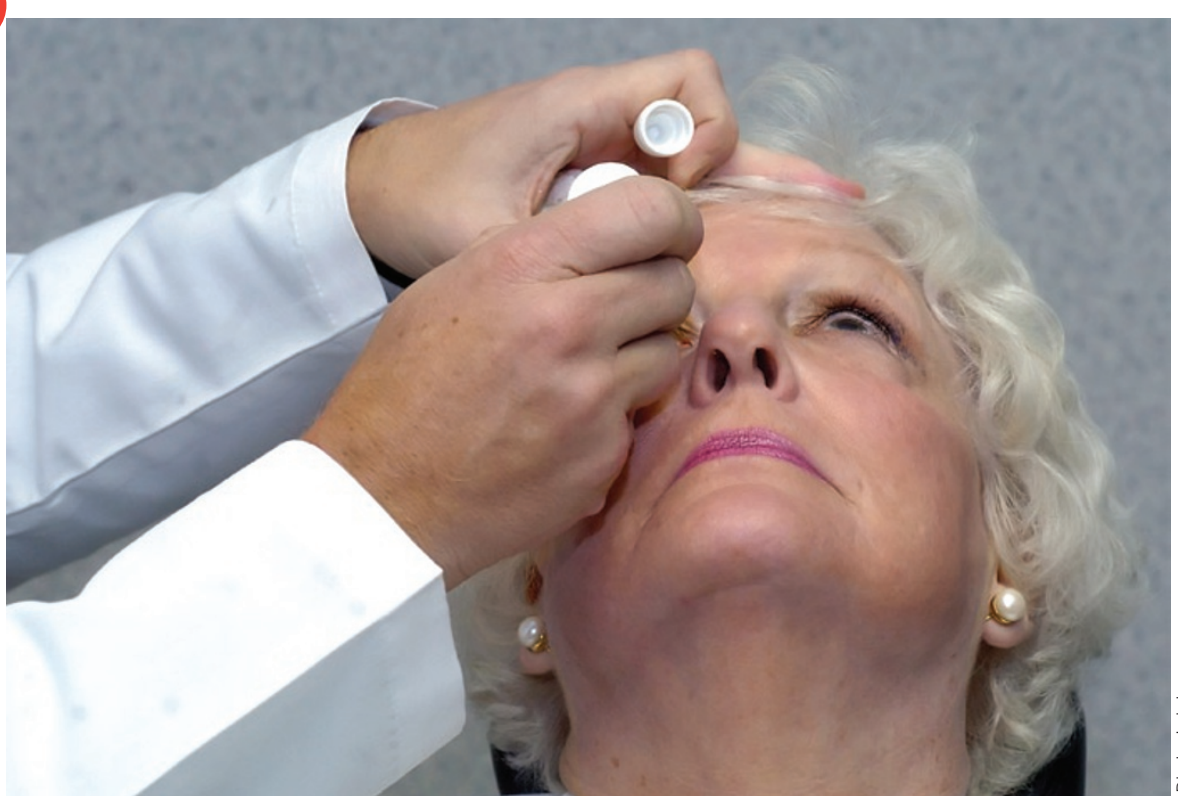

Improving sight: Doctors and companies don't always see eye to eye on which drug is best.

priced at $\$ 2,000$ a dose, or as much as $\$ 50,000$ for an entire course of treatment. In October, the company said that in 2008 it would stop supplying Avastin to compounding pharmacies, where ophthalmologists typically had the drug mixed for their patients. The company cited concerns over the sterility of drug processing at such pharmacies among its reasons for its plans to restrict distribution.

Although the Lucentis treatment costs 40 times more per dose than the Avastin treatment, Genentech thought it could convince doctors to switch to the new medication. After all, in previous cases, other drug companies had been able to persuade doctors to go along with them in somewhat similar situations. For instance, when the company AstraZeneca in 2001 introduced the heartburn medication Nexium, which was derived from a blockbuster drug about to go off patent, many doctors convinced their patients to switch to the new and nearly identical drug.

But Genentech bet wrong, perhaps not realizing that retinal specialists were fed up with the escalating cost of drugs in their field. In 2000, Novartis's Visudyne hit the market at $\$ 1,350$ per dose. Next came Pfizer's Macugen, approved in 2004 and priced at $\$ 1,000$ per dose, or about $\$ 13,500$ per case of the disease. So that same year, when Avastin became available, it was a breakthrough — not only was it incredibly effective, but it was cheap, too: "It was revolutionary, like penicillin," says Greg Rosenthal, a retinal specialist in Toledo, Ohio. 